\title{
Bioclimate and diversity of Tamaricaceae in Oranie, West Algeria
}

\section{Mohammed Souddi ${ }^{1,2}$, Bahae-Ddine Ghezlaoui-Bendi-Djelloul ${ }^{1 *}$}

${ }^{1}$ Laboratory of Ecology and Management of the Natural Ecosystems $\mathrm{N}^{\circ} 13$, Faculty of Nature and Life Sciences, and Earth and the Universe Sciences, University of Tlemcen, Algeria

${ }^{2}$ Department of Nature and Life Sciences, Faculty of Sciences and Technology, University of Adrar, Algeria

${ }^{\star}$ Corresponding author, E-mail: ghezlaouibahae@gmail.com

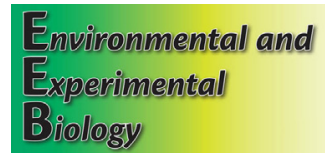

ISSN 2255-9582

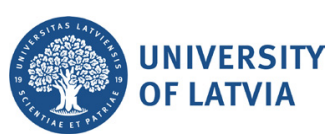

\begin{abstract}
Vegetation is a good indicator of the status of ecosystems that adapt to environmental changes. In this study, the diversity, floristic composition of Tamaricaceae and distribution of plant species under climate change were determined in West Algeria. Four sites were selected for performing phytoecological surveys using the sigmatist method along a north-south ecological gradient. In total, 195 plant species belonging to 41 families were identified, dominated by Asteraceae (15.90\%) and Poaceae (15.38\%). Analysis of biological types showed dominance of therophytes $(49.74 \%)$ in this flora, which indicates the presence of therophytization in this region. Analysis of chorological types identified the dominance of the Mediterranean element (46.67\%). The disturbance index was relatively high (75.54\%) reflecting significant importance of disturbance in the formation of the characteristic vegetation of the region. The study concluded that the flora of Tamaricaceae is under the threat of climate change and anthropogenic pressure. These results underline the need for strict policies to maintain and conserve the ecosystems that are vulnerable.
\end{abstract}

Key words: climate, diversity, Oranie, Tamaricaceae, west Algeria. Abbreviations: WBCS, Worldwide Bioclimatic Classification System.

\section{Introduction}

Tamarix L. is one of the largest genus in the family Tamaricaceae and is represented by 55 species worldwide (Heywood et al. 2007). Tamarix is native to the Mediterranean countries, former Soviet Union, China, India, North Africa, and southern Africa (Baum 1978; Heywood et al. 2007). The family Tamaricaceae is divided into five genera: Hololachna Ehrenb., Myricaria Desv., Reaumuria L., Tamarix L. and Myrtama Ovcz. and Kinzik (Crin 1989). In Algeria, 10 species of Tamarix have been reported: Tamarix gallica, Tamarix africana, Tamarix aphylla, Tamarix boveana, Tamarix pauciovulata, Tamarix parviflora, Tamarix brachystylis, Tamarix anglica, Tamarix speciosa, and Tamarix balansae (Quezel, Santa 1962; Quezel, Santa 1963).

Species of the Tamaricaceae in Algeria are found in arid and semi-arid environments that play a major role in the socioeconomic life of the local rural population. The species of Tamarix are used in animal feed, especially during the dry period, and as ornamental plants, they reduce desertification and have been used in traditional medicine (Villar et al. 2014). Thus, it is very important to study their diversity and species composition.
The study of floristic biodiversity, its spatial and temporal distribution and its interaction with the environment is very essential for the valuation and preservation of the natural ecosystems. In the global context of preserving biodiversity, the study of the flora of the Mediterranean basin presents great interest given the diversity of the historical, paleogeographical, paleoclimatic, ecological and geological factors that determine the secular impact of human pressure (Quezel et al. 1980).

In the region of Oranie, halophyte vegetation plays a strategic role both in pastoral and environmental terms; it constitutes a vast heritage by the diversity of ecological systems it integrates (Ghezlaoui 2011). Various studies have been conducted to assess halophyte diversity in western Algeria (Merzouk 2010; Sari-Ali et al. 2011), and, in particular, on the Tamarix in the region of Tlemcen (Bemmoussat 2004; Ghezlaoui 2011; Hadj-Allal 2013; Belkhoudja 2014). The aim of the present study was to obtain data about the species composition and diversity of Tamaricaceae, and to characterize the ecological gradients that govern their diversity and distribution in the region of Oranie in western Algeria. Because of the influence of the climate on plant composition, a climatic analysis updated by a global bioclimatic classification (Rivas Martinez 1993; 2004) was applied to the region. 


\section{Materials and methods}

\section{Study area and field sampling}

The study area was located in northwestern Algeria between $0^{\circ} 32^{\prime}$ to $02^{\circ} 00^{\prime} \mathrm{W}$ and $34^{\circ} 30^{\prime}$ to $35^{\circ} 49^{\prime} \mathrm{N}$. Administratively, it is located at the junction of the provinces of Tlemcen and Aïn Témouchent (Fig. 1).

Sampling was performed in 2014 to 2017 during spring, a period corresponding to optimum vegetation development in the studied area. In total, 70 phytoecological surveys at four sites were performed, which were chosen according to the sigmatist method (Braun-Blanquet 1952).

Site 1 (Rachgoun) was located at the junction of the National Route $\mathrm{N}^{\circ} 22$, which links Rachgoun to Beni-Saf. It was characterized by diverse vegetation formations with the presence of Tamarix gallica next to Oued Tafna and the presence of other plant species (Atriplex halimus, Malva sylvestris, Pistacia lentiscus, Quercus ilex, and Withania frutescens).

Site 2 (Remchi) was located north-west of Remchi. It was characterized by the presence of Tamarix gallica and Atriplex halimus.

Site 3 (Hammam Boughrara) was located $3 \mathrm{~km}$ east of the village of Hammam Boughrara. The site was dominated by Tamarix gallica, Atriplex halimus, and Salsola vermiculata.

Site 4 (El-Aouedj) was located southwest of the province of Tlemcen in the steppe zone. It was characterized by the presence of Tamarix boveana in the form of reforestation.

\section{Data collection and plant identification}

At each site, floristic surveys were carried out on a floristic homogeneous surface (Guinochet 1973). For each survey, floristic, geographical and environmental data were collected. The floristic surveys were carried out using the "minimal area" approach adapted to the type of vegetation. The minimum area of the stations varied between $64 \mathrm{~m}^{2}$ (El-Aouedj) and $100 \mathrm{~m}^{2}$ (Rachgoun, Remchi, Hammam Bougrhrara). This area varied significantly depending on the number of annual species present at the time of surveying and therefore precipitation and operating conditions (Djebaili 1984). Only the presence-absence of species was considered, as the objective was the discrimination and characterization of species inventoried in the study area (Ghezlaoui 2011). The botanical identification and classification into chorological types of the collected taxa was made using the flora of Quezel and Santa $(1962 ; 1963)$. The classification of plants according to their life form sensu Raunkiaer (1934) was based on the position of the perennating buds, but this classification was adjusted according to the local characteristics of the environment. The perturbation index was used to quantify therophytisation. It was calculated according to Loisel and Gamila (1993) as the ratio of the sum of chamaephytes and therophytes from the full number of the species.

\section{Bioclimatic synthesis}

Climate can exert direct and indirect effects on all components of ecosystems (Khatibi et al. 2016). The bioclimatic concept is wide and from an ecologic viewpoint shows the combination of elements that determine plant and animal life (Mankolli et al. 2009). While most systems of bioclimatic classification depend on limited variables such as precipitation, temperature, and their combinations, describing the climate of a region requires the evaluation of more factors (Khatibi et al. 2016).

The present bioclimatic study was based on historical climatic data (1913 to 1938) that were obtained from the

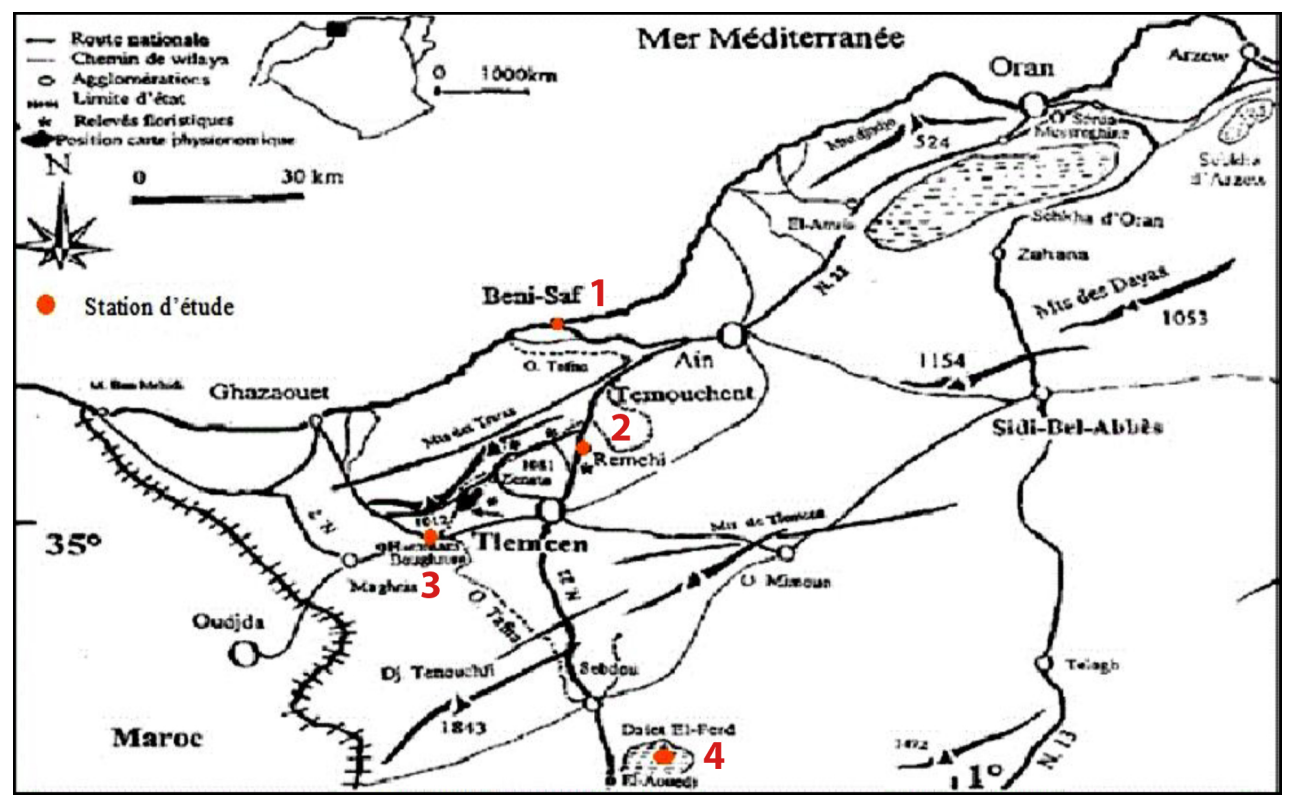

Fig. 1. Map of the study area with locations of study sites. 
Table 1. Geographical coordinates of reference meteorological stations

$\begin{array}{lcccc}\text { Station } & \text { Latitude (north) } & \text { Longitude (west) } & \text { Altitude (m) } & \text { Wilaya } \\ \text { Béni Saf } & 35^{\circ} 18^{\prime} & 1^{\circ} 21^{\prime} & 68 & \text { Ain-Temouchent } \\ \text { Zenata } & 35^{\circ} 01^{\prime} & 1^{\circ} 27^{\prime} & 249 & \text { Tlemcen } \\ \text { Hammam Boughrara } & 34^{\circ} 81^{\prime} & 1^{\circ} 47^{\prime} & 426.5 & \text { Tlemcen } \\ \text { Sebdou } & 32^{\circ} 42^{\prime} & 1^{\circ} 18^{\prime} & 1100 & \text { Tlemcen } \\ \text { El Aricha } & 34^{\circ} 12^{\prime} & 1^{\circ} 16^{\prime} & 1250 & \text { Tlemcen }\end{array}$

meteorological collection of Seltzer (1946) and recent meteorological reports that were taken from the National Office of meteorology from reference meteorological stations (Table 1). Many studies on climatology and bioclimatology have been carried out in Algeria in general and in the region of Tlemcen (Oranie) in particular (Benabadji, Bouazza 2000; Bestaoui 2001; Hasnaoui 2008; Merzouk 2010). All of these studies recognize that the Algerian climate belongs to the Mediterranean climate (Ghezlaoui, Benabadji 2018).

Recently, botanists and biogeographers have been using the bioclimatic classification system established by RivasMartinez (1981), known as the Worldwide Bioclimatic Classification System (WBCS). The WBCS is a bioclimatic classification system that is widely used in vegetation sciences, especially in geobotany and landscape ecology (Rivas-Martinez 1993). In this study, we applied the WBCS to five meteorological stations following the approach of Rivas-Martinez (1993; 2004).

The bioclimatic classification of the region of Tlemcen was derived by calculations of the bioclimatic indices necessary to define the bioclimate of each station. In particular, continentality, ombrotype and thermicity indices were obtained (Rivas-Martinez et al. 2011).

In the bioclimatic classification of Oranie, the simple continentality index was used. This continentality index expresses the difference between the average temperature of the hottest month and that of the coldest month of the year (Rivas-Martínez et al. 2011).

The thermicity index $\left(I_{t}\right)$ is the sum of the mean annual temperature, mean minimum temperature of the coldest month and mean maximum temperature coldest monthly period multiplied by 10 (Rivas-Martínez et al. 2004a).

The compensated thermicity index $\left(I_{t c}\right)$ was proposed to summarize the thermic constraints of climate to plant growth:

$$
I_{t c}=(T+m+M) \times 10+C,
$$

where $T$ is the mean annual temperature $\left({ }^{\circ} \mathrm{C}\right), m$ is the mean minimum temperature of the coldest month of the year $\left({ }^{\circ} \mathrm{C}\right), M$ is the average maximum temperature of the coldest month of the year $\left({ }^{\circ} \mathrm{C}\right)$, and $C$ is a compensation parameter, used if the climate is too continental or too oceanic, so that the $I_{t c}$ values for any part of the world can be significantly compared (Rivas-Martínez et al. 2004 b).

The annual ombrothermic index $\left(I_{0}\right)$ aims at expressing the average annual availability of water to plants, by including in its formulation the reduction in rainfall efficiency with increasing temperatures:

$$
I_{o}=P_{p} / T_{p}
$$

where $P_{p}$ is the total rainfall $(\mathrm{mm})$ of all months with positive average temperature and $T_{p}$ is the sum of the monthly average temperature of all months with positive average $\left({ }^{\circ} \mathrm{C}\right)$ (Mesquita, Sousa 2009).

Positive temperature $\left(T_{p}\right)$ is again a thermic index, used for defining thermotypes thresholds when the temperatures are particularly low:

$$
T_{p}=\sum T_{i}
$$

where $T_{i}$ is the monthly average temperature of any month with positive average temperature in tenths of degrees Celsius (Mesquita, Sousa 2009).

\section{Multivariate analysis}

Vegetation analysis was performed by correspondence analysis using Minitab 16 software. The variables were introduced in a form of codes in order to facilitate the reading of output ordinations. These codes were represented by lowercase letters taken from the vernacular name of the taxa present and identified from the flora of Quezel and Santa (1962). For example, species Tamarix gallica was assigned the code (Ta ga) (Ghezlaoui, Benabadji 2018). Presence and absence data were used in the correspondence analysis.

Two-dimensional (Axis 1 - 2) species ordinations were produced and eigenvalues and inertia values.

\section{Results and discussion}

\section{Bioclimatic synthesis}

The climatic characteristics at the meteorological stations used in this study are presented in Table 2. The Mediterranean macrobioclimate was the dominant pattern, with a wide range of bioclimate variation that included Mediterranean pluviseasonal-oceanic, Mediterranean xeric-oceanic and Mediterranean xeric continental types. Four thermotypic horizons and three ombrotypic horizons were identified (Table 2).

Almost all the meteorological stations in the Tlemcen region had an oceanic xeric Mediterranean bioclimate for both periods, which was characterized by high continentality, low rainfall during the growing season or months with average temperature $>0{ }^{\circ} \mathrm{C}$, and long dry season duration of 4 to 12 months (Rivas-Martínez et 
Table 2. Climatic data of the study area in the reference meteorological stations. $\mathrm{T}$, mean annual temperature; $\mathrm{T}_{\min }$, minimum temperature; $\mathrm{T}_{\text {max }}$, maximum temperature; $\mathrm{P}_{\mathrm{p}}$, total rainfall $(\mathrm{mm})$ of all months with positive average temperature; $\mathrm{I}_{\mathrm{c}}$, thermicity index; $\mathrm{I}_{\mathrm{tc}}$, compensated thermicity index; Io, annual ombrothermic index; $\mathrm{T}_{\mathrm{p}}$, sum of the monthly average temperature of all months with positive average

\begin{tabular}{|c|c|c|c|c|c|c|c|c|c|c|c|c|}
\hline Station & Year & $\mathrm{T}\left({ }^{\circ} \mathrm{C}\right)$ & $\mathrm{T}_{\min }$ & $\mathrm{T}_{\max }$ & $P_{p}$ & $I_{c}$ & $I_{\text {tc }}$ & $I_{0}$ & $T_{p}$ & Bioclimate & Ombrotype & Thermotype \\
\hline \multirow[t]{2}{*}{ Béni Saf } & $\begin{array}{c}1913- \\
1938\end{array}$ & 18.15 & 9.10 & 29.30 & 371.00 & 20.2 & 565.5 & 1.70 & 217.80 & $\begin{array}{l}\text { Mediterranean } \\
\text { xeric-oceanic }\end{array}$ & $\begin{array}{l}\text { Semiarid } \\
\text { upper }\end{array}$ & $\begin{array}{l}\text { Infra mediter- } \\
\text { ranean lower }\end{array}$ \\
\hline & $\begin{array}{c}1980- \\
2013\end{array}$ & 18.93 & 10.34 & 30.30 & 389.07 & 19.96 & 595.7 & 1.71 & 227.16 & $\begin{array}{l}\text { Mediterranean } \\
\text { xeric-oceanic }\end{array}$ & $\begin{array}{l}\text { Semiarid } \\
\text { upper }\end{array}$ & $\begin{array}{c}\text { Thermotropical } \\
\text { upper }\end{array}$ \\
\hline \multirow[t]{2}{*}{ Zenata } & $\begin{array}{c}1913- \\
1938\end{array}$ & 15.91 & 6.70 & 32.00 & 474.00 & 25.3 & 546.1 & 2.48 & 190.92 & $\begin{array}{c}\text { Mediterranean } \\
\text { pluviseasonal } \\
\text { oceanic }\end{array}$ & Dry lower & $\begin{array}{l}\text { Infra mediter- } \\
\text { ranean lower }\end{array}$ \\
\hline & $\begin{array}{c}1980- \\
2013\end{array}$ & 19.95 & 5.47 & 33.06 & 340.57 & 27.59 & 584.8 & 1.42 & 239.40 & $\begin{array}{c}\text { Mediterranean } \\
\text { xeric-continental }\end{array}$ & $\begin{array}{l}\text { Semiarid } \\
\text { lower }\end{array}$ & $\begin{array}{c}\text { Thermotropical } \\
\text { upper }\end{array}$ \\
\hline \multirow[t]{2}{*}{$\begin{array}{l}\text { Hammam } \\
\text { Boughrara }\end{array}$} & $\begin{array}{c}1913- \\
1938\end{array}$ & 16.82 & 3.00 & 26.96 & 418.00 & 23.96 & 467.8 & 2.07 & 201.84 & $\begin{array}{c}\text { Mediterranean } \\
\text { xeric-continental }\end{array}$ & Dry lower & $\begin{array}{l}\text { Infra mediter- } \\
\text { ranean upper }\end{array}$ \\
\hline & $\begin{array}{c}1977- \\
2013\end{array}$ & 17.09 & 3.46 & 34.89 & 297.28 & 31.43 & 554.4 & 1.45 & 205.08 & $\begin{array}{c}\text { Mediterranean } \\
\text { xeric-continental }\end{array}$ & $\begin{array}{l}\text { Semiarid } \\
\text { lower }\end{array}$ & $\begin{array}{l}\text { Infra mediter- } \\
\text { ranean lower }\end{array}$ \\
\hline \multirow[t]{2}{*}{ Sebdou } & $\begin{array}{c}1913- \\
1938\end{array}$ & 16.71 & 3.80 & 36.70 & 326.00 & 32.90 & 572.1 & 1.62 & 200.52 & $\begin{array}{c}\text { Mediterranean } \\
\text { xeric-continental }\end{array}$ & $\begin{array}{l}\text { Semiarid } \\
\text { upper }\end{array}$ & $\begin{array}{l}\text { Infra mediter- } \\
\text { ranean lower }\end{array}$ \\
\hline & $\begin{array}{c}1981- \\
2011\end{array}$ & 23.77 & 3.97 & 36.80 & 300.5 & 32.83 & 645.5 & 1.05 & 285.24 & $\begin{array}{c}\text { Mediterranean } \\
\text { xeric-continental }\end{array}$ & $\begin{array}{l}\text { Semiarid } \\
\text { lower }\end{array}$ & $\begin{array}{c}\text { Thermotropical } \\
\text { lower }\end{array}$ \\
\hline \multirow[t]{2}{*}{ El Aricha } & $\begin{array}{c}1913- \\
1938\end{array}$ & 13.67 & -1.50 & 35.60 & 296.80 & 37.10 & 477.7 & 1.81 & 164.04 & $\begin{array}{c}\text { Mediterranean } \\
\text { xeric-continental }\end{array}$ & $\begin{array}{l}\text { Semiarid } \\
\text { upper }\end{array}$ & $\begin{array}{l}\text { Infra mediter- } \\
\text { ranean upper }\end{array}$ \\
\hline & $\begin{array}{c}1984- \\
2009\end{array}$ & 14.57 & 0.00 & 32.94 & 198.00 & 32.94 & 475.1 & 1.13 & 174.84 & $\begin{array}{c}\text { Mediterranean } \\
\text { xeric-continental }\end{array}$ & $\begin{array}{l}\text { Semiarid } \\
\text { lower }\end{array}$ & $\begin{array}{l}\text { Infra mediter- } \\
\text { ranean upper }\end{array}$ \\
\hline
\end{tabular}

al. 1997). The exception was Zenata station, which had a bioclimatic Pluvi seasonal Mediterranean-continental, characterized by great continentality, relatively high rainfall during the growing season or months with average temperature $>0{ }^{\circ} \mathrm{C}$, and at least two consecutive dry summer months (Rivas-Martínez et al. 1997). This type of climate results in lower plant cover and floristic diversity, and development of halophilic vegetation (Tamaricaceae). Halophytes can also inhabit drier bioclimatic environments (Benabadji, Bouazza 2000).

\section{Floristic composition}

In total, 195 taxa at the four sites were recorded (Table 3). This richness was similar to that in the region of Tlemcen (Ghezlaoui, Benabbadji 2018). The taxa belonged to 42 botanical families. The most represented families were Asteraceae (31 taxa, 15.9\%), Poaceae (30 taxa, 15.38\%), Chenopodiaceae (15 taxa, 7.69\%) and Lamiaceae with a total of 13 taxa (6.67\%) (Fig. 2). Other families like Brassicaceae, Fabaceae and Liliaceae were moderately represented with 11, 11 and eight taxa respectively. The other families were poorly represented and accounted less than seven taxa. Asteraceae, Poaceae and Chenopodiaceae species are adapted to the arid and semi-arid environment in the Mediterranean regions (Ozenda 1983). In Oranie, a study on the floristic composition of the halophilic and salt-resistant plant population mention, dominance of the these plant families was also observed in the region of Hammam-Boughrara (Sari-Ali et al. 2012).

\section{Biological types}

The abundance of species by Raunkiaer life form (1934) was as follows: therophytes $>$ chamaephytes $>$ hemicryptophytes $>$ phanerophytes $>$ geophytes, with a clear predominance of therophytes (97 species, or 49.74\%; Fig. 3). Indeed, the dominance of therophytes indicates resistance to dry periods at high temperatures and is characteristic of Mediterranean vegetation in arid and semi-arid zones (Quezel 2000). It is thought that therophytes have a strategy of adaptation to adverse conditions by resistance to climatic extremes like summer drought in the Mediterranean context (Daget 1980; Madon, Medail 1997). Chamaephytes were the second most abundant life form with 49 species (25.13\%). The prevalence of chamaephytes can be explained by good adaptation to arid conditions (Raunkiaer 1934; Floret et al. 1990). Grazing by animals favours establishment of species that are not grazed by the herds (Benabadji et al. 2004). Hemicryptophytes came in third place with $10.77 \%$, which can be explained by low content of organic matter in soil (Le Houerou 1979). Phanerophytes and geophytes are less represented in the study area with respectively $7.69 \%$ and $6.67 \%$ of species, which could be explained by arid climatic conditions of the study area and the structural instability of the soil, which favours the development of species with 
Table 3. List of plant species inventoried in the study area with their families, biological types, and biogeographical types

\begin{tabular}{|c|c|c|c|c|}
\hline Families & $\begin{array}{l}\text { Species according to Quezel and Santa } \\
(1962-1963)\end{array}$ & Code & $\begin{array}{l}\text { Biological } \\
\text { type }\end{array}$ & Biogeographical type \\
\hline \multirow[t]{21}{*}{ Asteraceae } & Artemisia herba-alba Asso. & Art her & $\mathrm{CH}$ & Asie-occid \\
\hline & Astragalus pentaglottis L. & As pe & $\mathrm{TH}$ & Méd \\
\hline & Asteriscus maritimus (L). Less & As ma & $\mathrm{TH}$ & Canaries.Eu. Mérid.N.A \\
\hline & Atractylis cancellata $(\mathrm{L})$. & At ca & $\mathrm{CH}$ & Circum-méd. \\
\hline & Atractylis humilis L. & At hu & $\mathrm{HE}$ & Ibéro-Maur \\
\hline & Atractylis serratuloides Sieb. & At se & $\mathrm{CH}$ & Sah \\
\hline & Bellis annua $\mathrm{L}$. & Be an & $\mathrm{HE}$ & Circum-Méd \\
\hline & Bellis silvestris $\mathrm{L}$. & Be sy & $\mathrm{TH}$ & Circum-Méd \\
\hline & Calendula arvensis $\mathrm{L}$. & $\mathrm{Ca}$ ar & $\mathrm{TH}$ & Sub-Med \\
\hline & Centaurea pullata L. & Ce pu & $\mathrm{TH}$ & Méd \\
\hline & Chrysanthemum coronarium $\mathrm{L}$. & Ch co & $\mathrm{TH}$ & Méd \\
\hline & Chrysanthemum grandiflorum (L.) Batt. & Ch gr & $\mathrm{TH}$ & End \\
\hline & Echinops spinosus L. & Ec sp. & $\mathrm{CH}$ & Sub. Méd. Sah. \\
\hline & Evax pygmaea (L.) Brot. & Ev py & $\mathrm{TH}$ & Circum méd \\
\hline & Filago spathulata Presl & Fi sp & $\mathrm{TH}$ & Méd \\
\hline & Galactites tomentosa (L.) Moench & Ga to & $\mathrm{CH}$ & Circum bor \\
\hline & Launaea resedifolia O.K. & La re & $\mathrm{HE}$ & Méd.-Sah-Sind \\
\hline & Micropus bombicinus Lag. & Mi bo & $\mathrm{TH}$ & Euras. N.A. Trip \\
\hline & Pallenis spinosa (L.).Cass. & Pa sp & $\mathrm{CH}$ & Eur-Méd \\
\hline & Scorzonera undulate Vahl. & Sc un & $\mathrm{HE}$ & Méd \\
\hline & Taraxacum microcephalum Pomel. & Ta mi & $\mathrm{TH}$ & Méd \\
\hline \multirow[t]{21}{*}{ Poaceae } & Aegilops triuncialis $\mathrm{L}$. & Ae tr & $\mathrm{TH}$ & Méd-Irano-Tour \\
\hline & Agropyrum repens (L.) P.B. p.p. & Ag re & $\mathrm{TH}$ & Circum-bor \\
\hline & Ampelodesma mauritanicum (Poiret) Dur. et Sch. & Am ma & $\mathrm{CH}$ & W.Méd \\
\hline & Anagallis arvensis $\mathrm{L}$. & An ar & $\mathrm{TH}$ & Sub-Cosmop \\
\hline & Avena alba Vahl & Av al & $\mathrm{TH}$ & Méd-Iran-Tour \\
\hline & Avena sterilis L. & Av st & $\mathrm{TH}$ & Macar-Méd-Irano-Tour \\
\hline & Brachypodium distachyum (L.) P.B. & Br di & $\mathrm{TH}$ & Paleo-sub-Trop \\
\hline & Bromus rubens $\mathrm{L}$. & $\mathrm{Br} r u$ & $\mathrm{TH}$ & Paleo-sub-Trop \\
\hline & Cistus albidus L. & $\mathrm{Ci}$ al & $\mathrm{CH}$ & Méd \\
\hline & Echinaria capitata (L.) Desf. & Ec ca & $\mathrm{TH}$ & Atl - Méd \\
\hline & Glyceria fluitans (L.) R. Br. & Gl fl & $\mathrm{TH}$ & Sub-Cosmop \\
\hline & Hordeum murinum L. & Ho mu & $\mathrm{TH}$ & Circum bor \\
\hline & Koeleria phleoides (Vill.) Pers. & Ko ph & $\mathrm{TH}$ & Sub - cosm \\
\hline & Koeleria pubescens (Lamk.) P. B. & Ko pu & $\mathrm{HE}$ & W. Méd \\
\hline & Lygeum spartum L. & Ly sp & GE & W.Méd. \\
\hline & Phalaris bulbosa L. & $\mathrm{Ph}$ bu & $\mathrm{TH}$ & Macar-Méd \\
\hline & Poa bulbosa $\mathrm{L}$. & Po bu & $\mathrm{TH}$ & Paléo -Temp \\
\hline & Puccinellia maritima (Huds.) Parl. & Pu m & $\mathrm{HE}$ & Euras \\
\hline & Schismus barbatus (L.) TheIl. & Sc bar & $\mathrm{TH}$ & Macar-Méd \\
\hline & Stipa tenacissima L. & St te & GE & Ibero-Maur \\
\hline & Vulpia ciliata Link. & Vu ci & $\mathrm{CH}$ & Méd-Irano-Tour \\
\hline \multirow[t]{9}{*}{ Chenopodiaceae } & Arthrophytum scoparium (Pomel) Iljin & Ar sc & $\mathrm{CH}$ & Sah -Med \\
\hline & Atriplex dimorphostegia Kar. et Kir. & At di & $\mathrm{CH}$ & Sah-Sind. \\
\hline & Atriplex halimus L. & At ha & $\mathrm{CH}$ & Cosmop \\
\hline & Beta macrocarpa Guss. & Be ma & GE & Méd \\
\hline & Chenopodium album $\mathrm{L}$. & $\mathrm{Ch}$ al & $\mathrm{TH}$ & Cosmop \\
\hline & Halogeton sativus (L.) Moq. & Ha sa & $\mathrm{TH}$ & W.Méd. \\
\hline & Noaea mucronata (Forsk) Asch et Schw. & No mu & $\mathrm{CH}$ & Méd-Iran-Tour \\
\hline & Salsola foetida Del. & Sa fo & $\mathrm{TH}$ & Sah-Sind \\
\hline & Salsola vermiculata L. & Sa ver & $\mathrm{CH}$ & Sah-Méd \\
\hline
\end{tabular}


Table 3. continued

\begin{tabular}{|c|c|c|c|c|}
\hline Families & $\begin{array}{l}\text { Species according to Quezel and Santa } \\
(1962-1963)\end{array}$ & Code & $\begin{array}{l}\text { Biological } \\
\text { type }\end{array}$ & Biogeographical type \\
\hline Chenopodiaceae & Suaeda fruticosa $\mathrm{L}$. & Su fr & $\mathrm{CH}$ & Cosm \\
\hline \multirow[t]{8}{*}{ Lamiaceae } & Ballota hirsuta Pomel & Ba hi & $\mathrm{TH}$ & Ibéro-Maur \\
\hline & Lavandula dentata $\mathrm{L}$. & La de & $\mathrm{CH}$ & W.Méd \\
\hline & Lavandula multifida L. & La mu & $\mathrm{CH}$ & Méd \\
\hline & Salvia verbenaca (L.) Briq. & Sa ver & $\mathrm{HE}$ & Méd-Atl \\
\hline & Marrubium vulgare L. & Mar vu & $\mathrm{HE}$ & Cosmop \\
\hline & Teucrium polium subsp. capitatum L. & Te po & $\mathrm{CH}$ & Eur-Med \\
\hline & Teucrium pseudochamaepitys L. & Te ps & TH & W - Méd \\
\hline & Thymus ciliatus subsp coloratus Desf. & Th ci & $\mathrm{CH}$ & End. N.A \\
\hline \multirow[t]{9}{*}{ Brassicaceae } & Alyssum campestre L. & $\mathrm{Al} \mathrm{ca}$ & TH & Méd \\
\hline & Erucaria uncata (Boiss.) Asch. et Sch w. & Er un & TH & Sah-Sind. \\
\hline & Eruca vesicaria (L.) Car. & Er ve & HE & Méd \\
\hline & Lobularia maritima (L.) Desv. & Lo ma & TH & Méd \\
\hline & Matthiola longipetala (Vent.) OC. & Ma lo & $\mathrm{CH}$ & Méd.-Sah.-Sind \\
\hline & Muricaria prostrata (Desf.) Desv. & Mu pr & TH & End. N.A \\
\hline & Raphanus raphanistum $\mathrm{L}$. & Ra ra & $\mathrm{TH}$ & Méd \\
\hline & Sinapis arvensis $\mathrm{L}$. & $\mathrm{Si}$ ar & TH & Paléo-temp \\
\hline & Sisymbrium runcinatum Lag. & Si ru & TH & Méd.-Iran-Tour \\
\hline \multirow[t]{9}{*}{ Fabaceae } & Astragalus epiglottis L. & As ep & TH & Méd \\
\hline & Calycotome spinosa (L.) Lamk & Ca sp & $\mathrm{CH}$ & W.Méd \\
\hline & Medicago arborea $\mathrm{L}$. & Me ar & $\mathrm{CH}$ & Méd \\
\hline & Medicago minima Grufb. & Me mi & $\mathrm{TH}$ & Eur.-Méd \\
\hline & Medicago rugosa Desr. & Me ru & TH & Cosmp \\
\hline & Medicago truncatula Gaertn. & Me trun & TH & Méd \\
\hline & Trifolium angustifolium L. & $\operatorname{Tr}$ an & TH & Méd \\
\hline & Trifolium stellatum L. & Tr st & TH & Méd \\
\hline & Trigonella polycerata $\mathrm{L}$. & $\operatorname{Tr}$ po & $\mathrm{TH}$ & Ibéro-Maur \\
\hline \multirow[t]{5}{*}{ Liliaceae } & Asparagus acutifolius L. & As ac & GE & Méd \\
\hline & Asparagus albus L. & As al & GE & W.Méd \\
\hline & Asparagus stipularis Forsk. & As st & GE & Macar-Méd \\
\hline & Asphodelus microcarpus Salzm. et Viv. & As mi & GE & Canar-Méd \\
\hline & Urginea maritima (L.) Baker & Ur ma & GE & Canar.Méd. \\
\hline \multirow[t]{5}{*}{ Cistaceae } & Cistus albidus L. & $\mathrm{Ci}$ al & $\mathrm{TH}$ & Cosmop \\
\hline & Cistus villosus L. & Ci vi & $\mathrm{CH}$ & Méd \\
\hline & Helianthemum apertum Pomel & He ap & TH & End. N.A \\
\hline & Helianthemum cinereum subsp. rubellum (Presl.) M. & He ci & $\mathrm{HE}$ & Eur. Mérid (sauf france). N.A \\
\hline & Helianthemum hirtum Mill. & He hi & $\mathrm{CH}$ & N.A \\
\hline \multirow[t]{4}{*}{ Apiaceae } & Bupleurum semicompositum L. & Bu se & $\mathrm{TH}$ & Méd \\
\hline & Daucus carota L. (sensu lato) & $\mathrm{Daca}$ & TH & Méd \\
\hline & Eryngium triscuspidatum L. & Er tr & TH & W.Méd \\
\hline & Thapsia garganica $\mathrm{L}$ & Th ga & $\mathrm{CH}$ & Méd \\
\hline \multirow[t]{3}{*}{ Plantaginaceae } & Plantago albicans $\mathrm{L}$. & $\mathrm{Pl}$ al & $\mathrm{TH}$ & Méd \\
\hline & Plantago lagopus L. & Pl la & $\mathrm{HE}$ & Méd \\
\hline & Plantago ovata Forssk. & $\mathrm{Pl}$ ov & $\mathrm{TH}$ & Méd \\
\hline \multirow[t]{3}{*}{ Zygophyllaceae } & Fagonia arabica L. & $\mathrm{Fa}$ ar & TH & Sah-Sind \\
\hline & Fagonia cretica L. & $\mathrm{Fa} \mathrm{cr}$ & TH & Méd \\
\hline & Peganum harmala L. & Pe ha & $\mathrm{CH}$ & Méd \\
\hline \multirow[t]{2}{*}{ Tamaricaceae } & Tamarix boveana Bunge & Ta bo & PH & Sah \\
\hline & Tamarix gallica L. & Ta ga & $\mathrm{PH}$ & N.Trop \\
\hline \multirow[t]{2}{*}{ Boraginaceae } & Echium pycnanthum subsp. humile (Desf.) Jah. et M. & Ec py & $\mathrm{TH}$ & Méd. Sah \\
\hline & Echium vulgare $\mathrm{L}$. & Ec vu & $\mathrm{HE}$ & Méd \\
\hline
\end{tabular}


Table 3. continued

\begin{tabular}{|c|c|c|c|c|}
\hline Families & $\begin{array}{l}\text { Species according to Quezel and Santa } \\
(1962-1963)\end{array}$ & Code & $\begin{array}{l}\text { Biological } \\
\text { type }\end{array}$ & Biogeographical type \\
\hline \multirow[t]{2}{*}{ Malvaceae } & Malva aegyptiaca L. & Ma ae & $\mathrm{CH}$ & Sah-sub-Med \\
\hline & Malva sylvestris $\mathrm{L}$. & Ma sy & $\mathrm{TH}$ & Euras \\
\hline \multirow[t]{2}{*}{ Euphorbiaceae } & Euphorbia exigua L. & Eu ex & $\mathrm{TH}$ & Méd. Eur \\
\hline & Euphorbia falcata L. & $\mathrm{Eu} \mathrm{fa}$ & $\mathrm{TH}$ & Méd. As \\
\hline \multirow{2}{*}{ Caryophyllaceae } & Herniaria hirsuta L. & He hi & $\mathrm{TH}$ & Paleo -Temp \\
\hline & Paronychia argentea (Pourr.) Lamk. & $\mathrm{Pa}$ ar & $\mathrm{CH}$ & Méd \\
\hline \multirow[t]{2}{*}{ Oxalidaceae } & Oxalis corniculata $\mathrm{L}$. & Ox co & $\mathrm{TH}$ & Cosmp \\
\hline & Oxalis pes-caprae $\mathrm{L}$. & Ox pe & $\mathrm{TH}$ & Méd \\
\hline \multirow[t]{2}{*}{ Ranunculaceae } & Adonis dentata Del. & Ad de & $\mathrm{TH}$ & Méd \\
\hline & Ceratocephalus falcatus (L.) Pers. & Ce fa & $\mathrm{TH}$ & Méd-Iran-Tour \\
\hline Anacardiaceae & Pistacia lentiscus L. & Pi le & $\mathrm{PH}$ & Méd \\
\hline Géraniaceae & Erodium moschatum (Burm.) L'Her. & Er mo & $\mathrm{TH}$ & Méd \\
\hline Pinaceae & Pinus halepensis L. & Pi ha & $\mathrm{PH}$ & Méd \\
\hline Convolvulaceae & Convolvulus althaeoïdes L. & Co al & $\mathrm{TH}$ & Macar-Méd \\
\hline Primulaceae & Anagallis arvensis $\mathrm{L}$. & An ar & $\mathrm{TH}$ & Sub-Cosmop \\
\hline Resedaceae & Reseda alba L. & Re al & $\mathrm{TH}$ & Euras \\
\hline Rhamnaceae & Ziziphus lotus (L.) Desf. & Zi lo & $\mathrm{PH}$ & Méd \\
\hline Thymeleaceae & Daphne gnidium L. & Da gn & $\mathrm{CH}$ & Méd \\
\hline Rosaceae & Sanguisorba minor Scop. & Sa mi & $\mathrm{TH}$ & Euras \\
\hline Globulariaceae & Globularia alypum L. & Gl al & $\mathrm{CH}$ & Méd \\
\hline Papaveraceae & Papaver rhoeas L. & Pa rh & $\mathrm{TH}$ & Paléo-Temps \\
\hline Apocynaceae & Nerium oleander L. & $\mathrm{Ne}$ ol & $\mathrm{CH}$ & Méd \\
\hline Oleaceae & Olea europea L. & $\mathrm{Ol} \mathrm{eu}$ & $\mathrm{PH}$ & Méd \\
\hline Rutaceae & Ruta chalepensis L. & Ru ch & $\mathrm{CH}$ & Méd \\
\hline Urticaceae & Urtica membranacea Poiret & Ur me & $\mathrm{TH}$ & Méd \\
\hline Solanaceae & Withania frutescens Pauquy & Wi fr & $\mathrm{CH}$ & Ibéro-Maur \\
\hline Myrtaceae & Eucalyptus globulus Labill. & Eu gl & $\mathrm{PH}$ & Aust-Méd \\
\hline Crassulaceae & Scabiosa stellata $\mathrm{L}$. & Sc st & $\mathrm{TH}$ & W.Méd \\
\hline Araliaceae & Hedera helix L. & He he & $\mathrm{TH}$ & Eur-Méd \\
\hline Mimosaceae & Acacia albida Del. & Ac al & PH & Af.Trop \\
\hline Palmaceae & Chamaerops humilis L. & Ch hu & $\mathrm{CH}$ & Méd \\
\hline Amaryllidaceae & Agave americana L. & $\mathrm{Ag}$ am & GE & Méd-Amér \\
\hline Fagaceae & Quercus ilex $\mathrm{L}$. & Qu il & $\mathrm{PH}$ & Méd \\
\hline
\end{tabular}

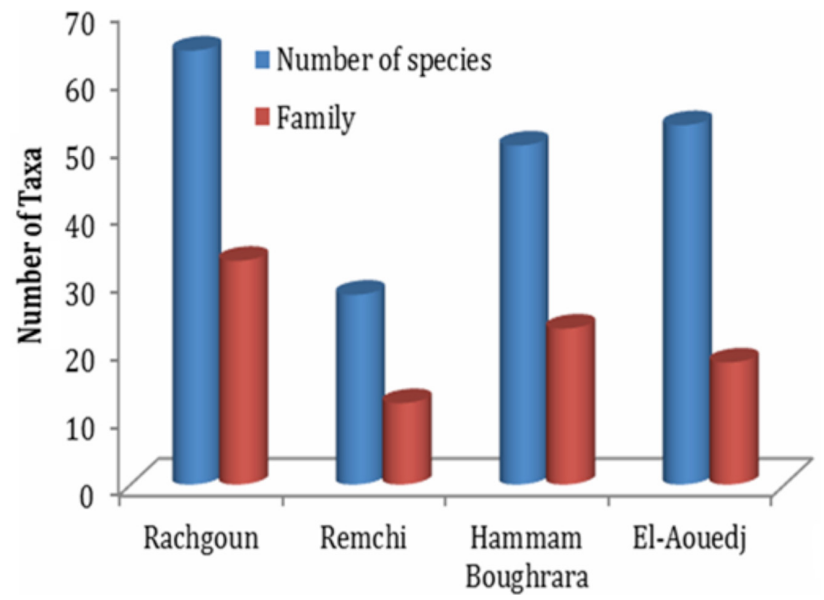

Fig. 2. Number of species and families in the different sites of the study area.

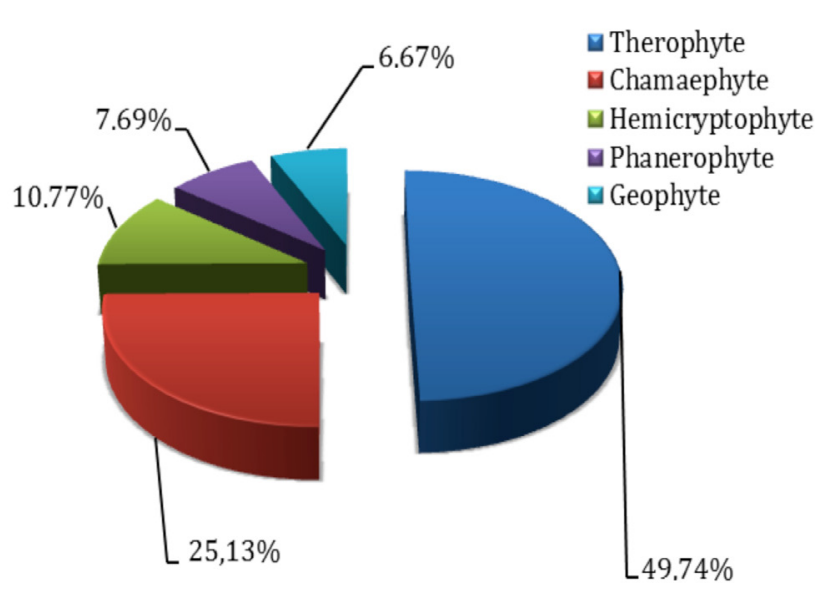

Fig. 3. Distribution of biological types in the study area. 


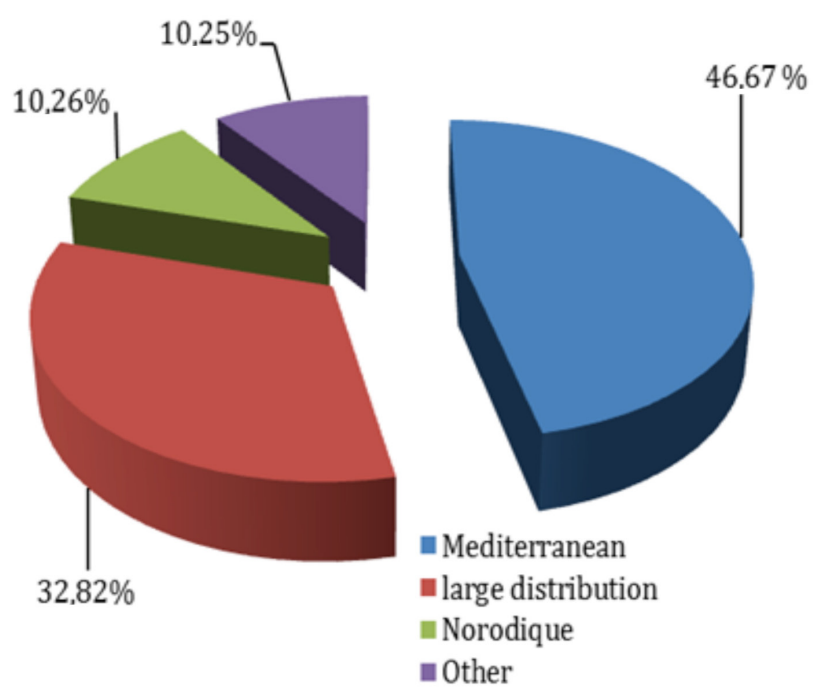

Fig. 4. Distribution of phytochoric elements in the study area.

a short life cycle and which are more or less demanding in terms of water and trophic needs (Ghezlaoui, Benabbadji 2018).

\section{Chorological types}

The analysis of the chorological spectrum of the region (Fig. 4) is generally marked by the predominance of the Mediterranean element (91 species, 46.67\%). This reflects the global and logical affinity of our flora to the Mediterranean region and its climatic conditions (Quezel 1983; Le Houerou 1995). Taxa with a large distribution were second most numerous with 64 taxa (32.82\%). The abundance of these species is generally related to therophytization of the Mediterranean flora caused by several ecological and anthropogenic factors (Miara et al. 2016). The Nordic taxa occupied the third position with a total number of 20 taxa (10.26\%). This low number can be explained by the remote geographical location of northern Europe (Habib et al. 2020). The other elements are poorly represented but contribute to the diversity of the plant genetic potential in the study area.

The perturbation index in the four sites of study was $75.54 \%$. This confirms the presence of therophytization in these regions by the dominance of therophytes and chamaephytes. Strong land degradation caused by man is clearly visible (clearing, grazing). Anthropogenic disturbances are largely responsible for the current vegetation structure in the Maghreb (Quezel, Barbero 1990). There have been many reports on disturbances caused by man and grazing, ranging from matorralisation through steppisation up to desertification (Berbero et al. 1990; Quezel 2000).

\section{Correspondence analysis}

Species ordinations (correspondence analysis) for the four sites are presented in Figs. 5 to 8, which show groups of species with similar ecological affinities (Aboura et al. 2006) to environmental factors (edaphic, nitrates, humidity) and form ecological gradients (Ghezlaoui et al. 2013). The first two axes explained most of the variation. For each ordination, the proportion of inertia (percentage variation explained) was calculated (Azzaoui et al. 2017).

Analysis of data for Rachgoun site is shown in Fig. 5 and Table 4 (value 2.30, inertia 22.61\%). Species at the top of the ordination included the post-cultural species (Sinapis arvensis) and halophyte (Tamarix gallica, Atriplex halimus). The presence of monospecific stands of Atriplex halimus

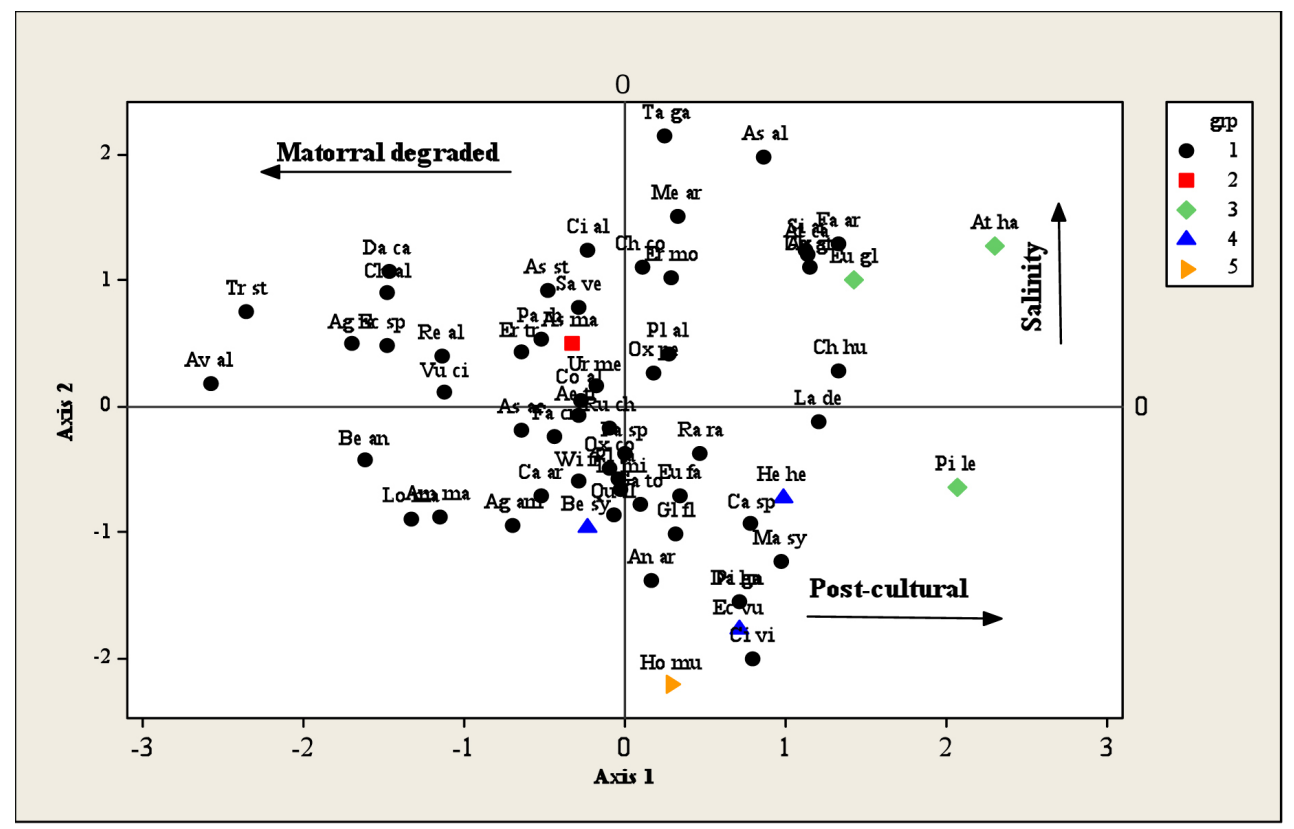

Fig. 5. Factorial plane of species Axis 1 - Axis 2 (Rachgoun site). 


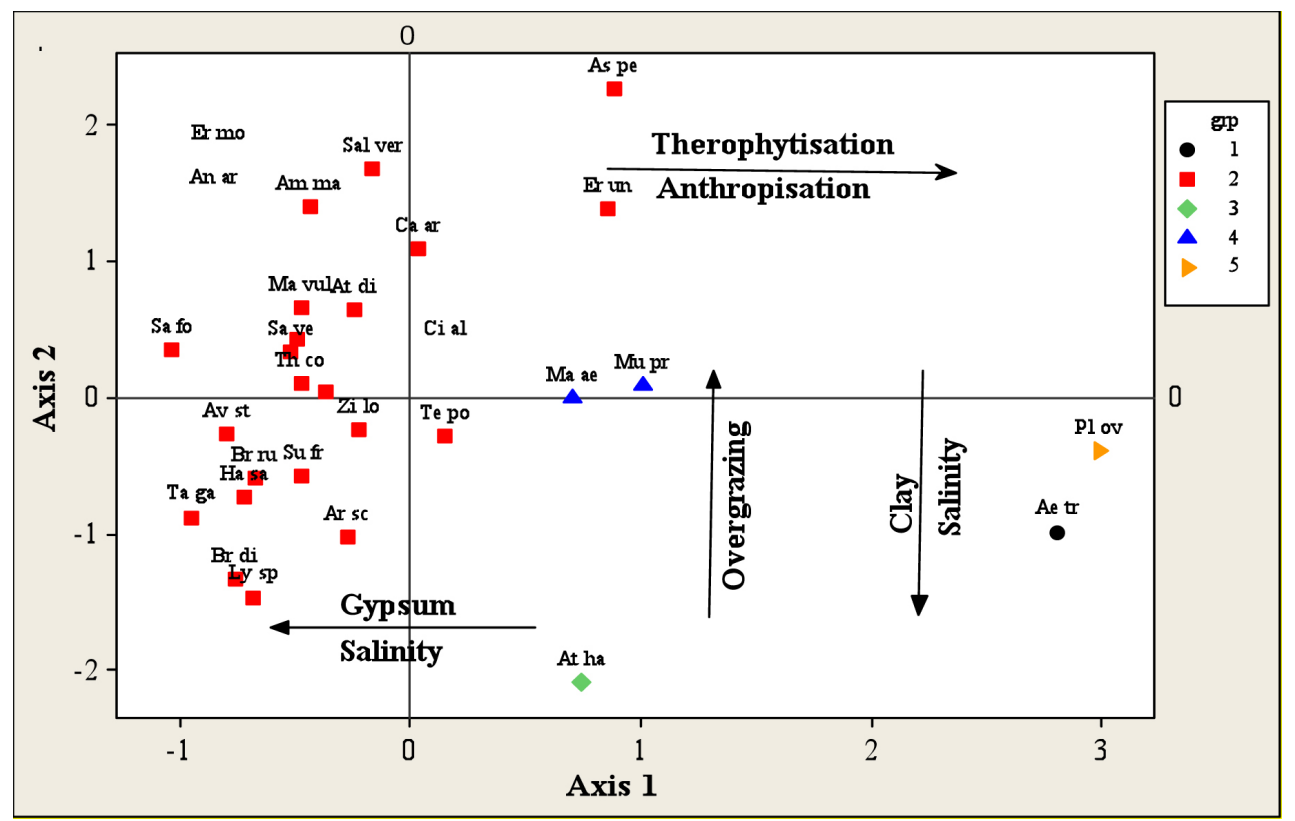

Fig. 6. Factorial plane of species Axis 1 - Axis 2 (Remchi site).

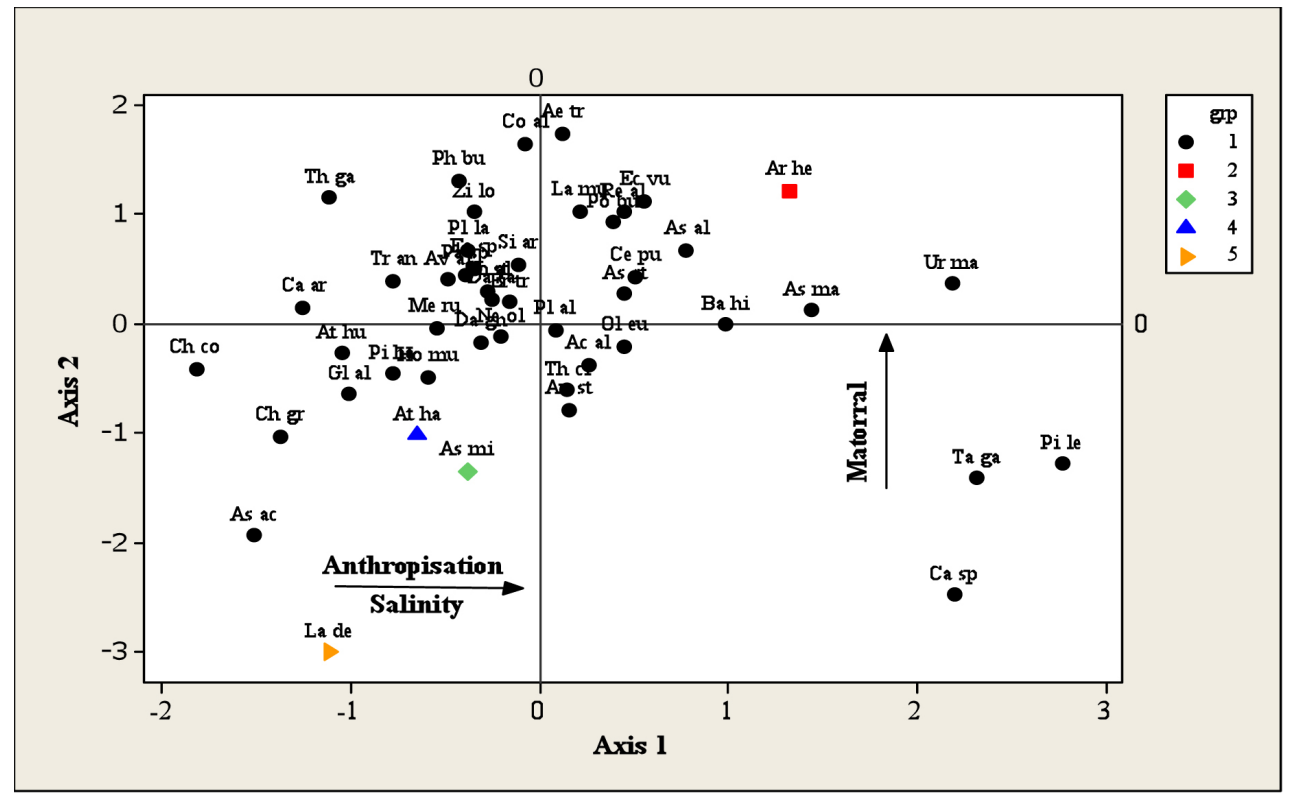

Fig. 7. Factorial plane of species Axis 1 - Axis 2 (Hammam Boughrara site).

or Tamarix gallica suggest higher salinity (Sari et al. 2012). Tamarix gallica is a very abundant plant, especially on the Mediterranean coast in beds of the river due to suitable moisture and salinity conditions (Ben-daânoun 1981). The presence of xerophyte species like Asparagus albus indicates anthropogenic action.

Pre-forest species such as Lobularia maritima were grouped on the bottom or left side of the ordination. Moreover, an increasing gradient of humidity from the top to bottom of the ordination was suggested by the presence of pre-forest species like Malva sylvestris at the bottom of the ordination.
Analysis of data for Remchi site is shown in Fig. 6 and Table 4 (value: 7.33 , inertia $36.7 \%$ ). This axis appeared to be characterized by a gradient of gypsum going from the right to left side of the ordination, and therophytisation the opposite direction.Terophytization can be explained by the ultimate stage of ecosystem degradation with subnitrophilic species associated with overgrazing and fertilizer residue (Barbero et al. 1981). Indeed, annual species (such as Plantago ovata, Astragalus sp., Pentaglottis sp.) represent the classes of Thero-Brachypodietea (Braun-Blanquet 1947) and Stellarietea mediae (Braun-Blanquet 1931).

Teucrium polium occurs in the centre of the ordination 


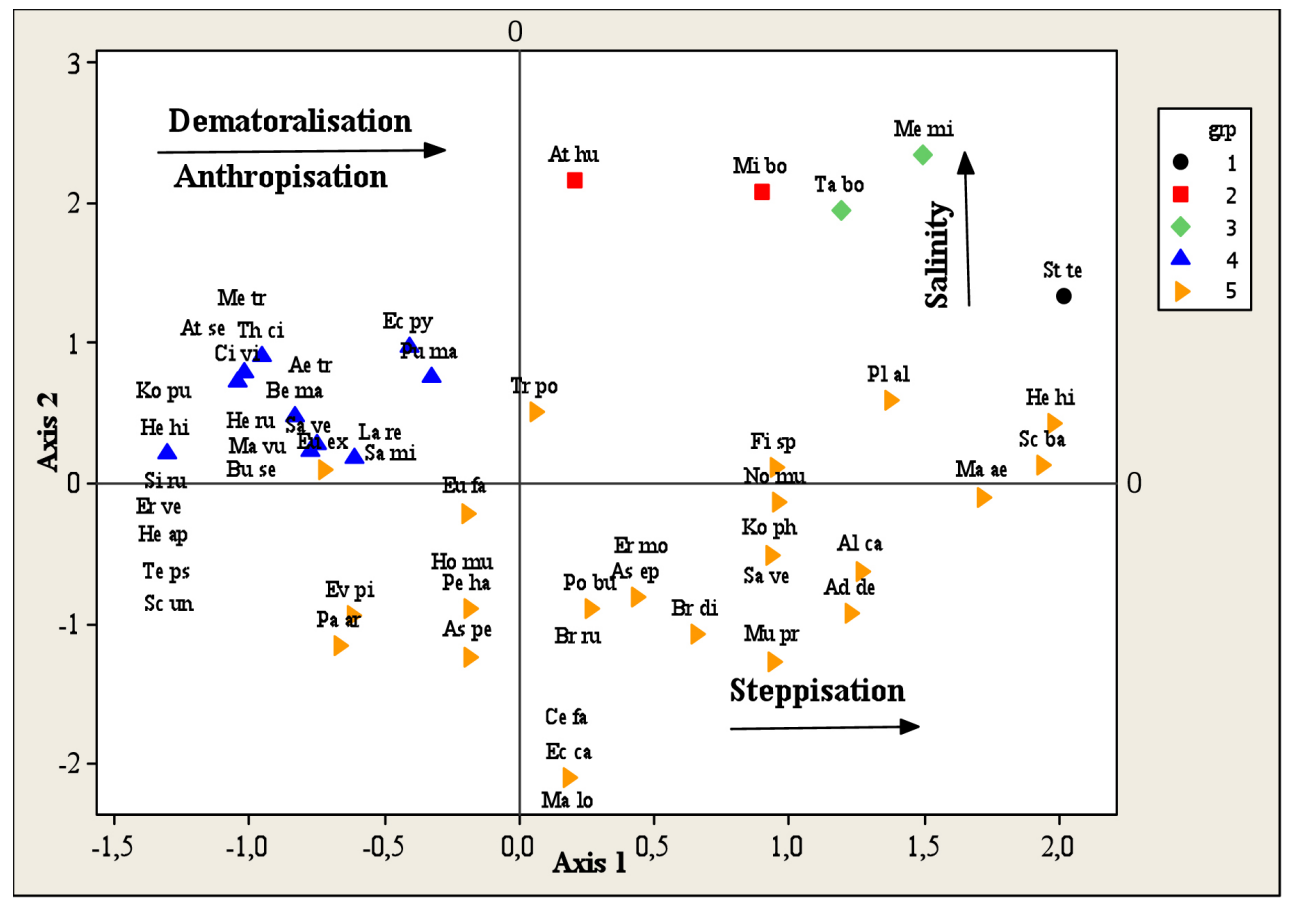

Fig. 8. Factorial plane of species Axis 1 - Axis 2 (El-Aouedj site).

and shows absolutely no affinity to any gradient. Halophyte species (such as Suaeda fruticosa, Salsola foetida) were grouped on the left side of the ordination and indicated an increasing salinity gradient to the left. These species are characteristic of Atriplico halimi-Suaedetum fruticosae ass. nov. (Aimé 1991) and Salsolo-Peganion alliance, which include mostly annual therophytes said nitratophiles (Sari-
Ali et al. 2012).

Analysis of data for Hammam Boughrara site is shown in Fig. 7 and Table 4 (value 2.55, inertia 22.60\%). The species Lavandula dentate and Asphodelus microcarpus were located at the bottom left of the ordination. These species are associated with low level of anthropization of the environment. Human action is also indicated

Table 4. High contribution taxa for the Axis 1 and Axis 2 of the factorial analysis of correspondence

\begin{tabular}{lll} 
Site & Positive pole & Negative pole \\
Rachgoun & Atriplex halimus $(2.29)$ & Avena alba $(-2.58)$ \\
& Sinapis arvensis $(1.12)$ & Trifolium stellatum $(-2.35)$ \\
& Malva sylvestris $(0.96)$ & Agave americana $(-0.69)$ \\
& Asparagus albus $(0.86)$ & Aegilops triuncialis $(-0.29)$ \\
& Tamarix gallica $(0.24)$ & Ampelodesma mauritanicum $(-0.15)$ \\
\hline Remchi & Plantago ovata $(2.99)$ & Salsola foetida $(-1.03)$ \\
& Aegilops triuncialis $(2.81)$ & Tamarix gallica $(-0.94)$ \\
& Astragalus pentaglottis $(0.88)$ & Lygeum spartum $(-0.67)$ \\
\hline Hammam Boughrara & Atriplex halimus $(0.74)$ & Suaeda fruticosa $(-0.46)$ \\
& Teucrium polium $(0.15)$ & Atriplex dimorphostegia $(-0.24)$ \\
& Urginea maritima $(2.18)$ & Thapsia garganica $(-1.10)$ \\
\hline Asteriscus maritimus $(1.44)$ & Lavandula dentate $(-1.10)$ \\
\hline Artemisia herba-alba $(1.33)$ & Atractylis humilis $(-1.04)$ \\
\hline Asparagus albus $(0.78)$ & Asphodelus microcarpus $(-0.38)$ \\
\hline Aegilops triuncialis $(0.12)$ & Atriplex halimus $(-0.33)$ \\
\hline Schismus barbatus $(1.93)$ & Teucrium pseudo-chamaepitys $(-1.30)$ \\
\hline Malva aegyptiaca $(1.71)$ & Koeleria pubescens $(-1.30)$ \\
\hline
\end{tabular}


by the presence of thorny and/or toxic species that are nonpalatable to cattle. The anthropization gradient from left to right was indicated by the presence of Asphodelus microcarpus, a non-palatable species on the left side of the ordination. The presence of Urginea maritima, an anthropozoic species, on the right side of the ordination indicated a particularly disturbed environment. This species is associated with ruderal communities that develop after prolonged overgrazing (Le Houerou (1995). Atriplex halimus, a perennial species with deep roots, has a great adaptation potential to develop in salt soils environments of very varied texture (Pouget 1980).

At the extreme left and below appear the species Scorzonera undulalta and lower still Teucrium chamaepitys species which occupies the stony slopes of the arid steppic or pre-steppic formations, indicates stable climax on substrates that are always highly saline and more often anthropogenic by intense grazing (Aimé 1991). They represent the ultimate state of dematoralization, which is trigger of steppization. Steppization causes a shift of vegetation from a forest to steppe type (Le Houerou 1995).

Analysis of data for the El-Aouedj site is shown in Fig. 8 and Table 4 (value 4.74, inertia 47.4\%). On the right side of the orination indicated salinity (Tamarix boveana) and overgrazing (Noaea mucronata) gradients. The species Noaea mucronata characterizes the steppe and pre-steppe vegetation and a state of advanced degradation. These communities are stable climaxes on substrates that are always quite saline and most often anthropized by intensive grazing (Aime 1991). Tamarix appears to be an exogenous species in the area (introduced by reforestation in the 1970s), as Simonneau (1952) notes that "the phanerophytes or trees are mostly planted. Some, however, are subspontaneous". Tamarix boveana communities have been undergoing significant regression under the combined effect of climate and anthropogenic pressure. Currently, the Tamarix vegetation occurs around the Daya (Ghezlaoui et al.2013).

On the left side of the ordination, there are steppe species of Mediterranean dry meadows (Thymus ciliatus, Koeleria pubescens). The gradients indicate anthropization related to cultivated plants (as Hordeum murinum) and therophitisation (as Astragalus pentaglottis, Marrubium vulgare). Thymus ciliatus subsp. coloratus is found on degraded soil with fine soil or limestone (Bouazza et al. 2001).

\section{Conclusions}

The application of the new global bioclimatic classification (Rivas-Martinez et al. 1993) in the study region made it possible to identify four thermotypic horizons and three ombrotypic horizons. The Mediterranean macrobioclimate was the dominant pattern, including a wide range of bioclimates such as the Mediterranean pluviseasonaloceanic, Mediterranean xeric-oceanic and Mediterranean xeric continental (Ghezlaoui, Benabadji 2018).

Tamaricaceae plant communities in western Algeria contained 195 species, dominated by the Asteraceae and Poaceae. The study area was characterized by important plant diversity and there was significant variation in specific richness along the North-South ecological gradients. The study area is threatened by climate change and human activity, which have caused degradation. The study of plant communities with the genus Tamarix in western Algeria, which was based on correspondence analysis, made it possible to characterize three ecological gradients responsible for the distribution of these taxa at the level of the four sites. These gradients correspond to the following ecological factors: salinity, moisture, anthropization and therophytization. It is important to take into account these gradients in order to succeed in conservative management in these ecosystems (Chemouri et al. 2015).

\section{Acknowledgements}

The Authors wish to pay a great tribute to Pr. Djamel Benmansour, professor in Department of Ecology and Environement (Faculty of Nature and life Sciences, and Earth and the Universe Sciences, University of Tlemcen, Algeria), who left us two years ago. His advice and guidance in data analysis has been of great help to us.

\section{References}

Aboura R. 2006. Phytoecological comparaison of Atriplexaie located in North and South of Tlemcen. Master Thesis. Tlemcen University, Algeria.

Aboura R., Benmansour D., Benabadji N. 2006. Phytoecological comparaison of Atriplexaie in en Oranie (Algeria). Ecol. Mediter. 32: 73-84.

Aime S. 1991. Ecological study of the transition between the sub-humid, semi-arid and arid populations in the thermo Mediterranean floor of the Oranian Tell (North-western Algeria). Ph.D. Thesis, Aix-Marseille III University, Marseille, France.

Azzaoui M.E., Maatoug M., Berrayah M. 2017. Phytoecological study of Tetraclinis articulata (Vahl) Mast.in the woodland of Beni Affene, Sdamas chergui (Tiaret, Algeria). J. Fund. Appl. Sci. 9: 971-987.

Barbero M., Quezel P., Rivas-Martinez S. 1981. Contribution to the study of forest and pre-forest groups in Morocco. Phytocoenologia 9: 311-412.

Babero M., Quezel P., Loisel R. 1990. Contributions of phytoecology in the interpretation of human induced changes and distibuances on Mediterranean ecosystems. Mediter. Forest 12: 194-216.

Baum B.R. 1978. The Genus Tamarix. Jerusalem Academic Press, Israel. 213 p.

Belkhoudja A. 2014. Diachronic study of formation at Tamaricaceae in the north of Tlemcen. Master Thesis. Tlemcen University, Algeria.

Benabadji N., Bouazza M. 2000. Contribution to a bioclimatic study of the steppe to Artemisia herba-alda Asso. in Oranie (Western Algeria). Dry Rev. 11: 117-123.

Benabadji N., Bouazza M., Metge G., Loisel R. 2004. The soils of 
the steppe at Artemisia herba-alba Asso. in south of Sebdou (Oranie, Algeria). Synthesis 13: 20-28.

Ben-daânoun M. 1981. Synecological and syndynamic study of the halophilic and hyrohalophilic vegetation of Bou-Regreg (Atlantic coast of Morocco). Application and development prospects. Ph.D. Thesis, Aix-Marseille III University, Marseille.

Bemmoussat F.Z. 2004. Bioclimatic and physionomic relationship of halophyte stands. Master Thesis. Tlemcen University, Algeria.

Bestaoui K. 2001. Contribution to a taxonomic and ecological study of matorrals in the region of Tlemcen. Magister Thesis. Tlemcen University, Algeria.

Bouazza M., Benabadji N., Loisel R. 2001. Assessment of the flora of Tlemcen region (Oranie - Algeria). Mediter. Forest 22: 130136.

Braun-Blanquet J. 1931. Overview of the lower Langue-doc vegetation. Sigma Communication 9: 35-40.

Braun-Blanquet J. 1947. The vegetation cover in the Montpellier region and its relationship with soil. Sigma Communication $\mathrm{N}^{\circ} 94$.

Braun-Blanquet J. 1952. Applied Phytosociology. Sigma Communication $\mathrm{N}^{\circ} 116$.

Chemouri F.Z., Ghezlaoui-Bendi-Djelloul B., Benabadji N. 2015. Phyto-diversity of the vegetation of the Tlemcen Mountains (Western Algeria). Rev. Ecol. Balkan. 7: 1-11.

Daget PH. 1980. On botanical biological types as an adaptative strategy (case of therophytes). In: Barbault R., Blandin P., Meyer J. (eds) Theoretical Ecology Research: Adaptative Strategies. $110 \mathrm{p}$.

Djebaili S. 1984. Phytosociological and phytoecological research on the vegetation of the high steppe plains and the Algerian Saharan Atlas. University Publications Office, Algiers. 177 p.

Floret C.H., Galan M.J., Le Floch H., Orshan G., Romane F. 1990. Growth forms and phenomorphology traits along an environmental gradient: tools for studying vegetation. J. Veget. Sci. 1: 71-80.

Ghezlaoui S.B.E. 2011. Biomorphology and polymorphism aerial devices of some halophytes in Oranie, case of Atriplex halimus L. and Tamarix gallica L.. Ph.D. Thesis, University of Tlemcen, Algeria.

Ghezlaoui S.B.E., Benabadji N., Benabadji N.2013. Phytoecological and phytoedaphological characterization of steppe plant communities in the south of Tlemcen (Western Algeria). Open J. Ecol. 3: 495-504.

Ghezlaoui S.B.E., Benabadji N. 2018. The vegetation of the Tlemcen mountains (Algeria). Phytoecological aspect. Bot. Complut. 42 : 101-124.

Guinochet M. 1973. Phytosociology. Ecology collection, Masson Ed., Paris. 227 p.

Habib N., Regagba Z., Miara M.D., Ait Hammou M., Snorek J. 2020. Floristic diversity of steppe vegetation in the region of Djelfa, North-West Algeria. Acta Bot. Malac. 45: http://dx.doi. org/10.24310/abm.v45i0.7987

Hadj Allal F. 2013. Contribution to the study of the genus Tamarix: Botanical and phytoecological aspects in the region of Tlemcen. Magister Thesis. Tlemcen University, Algeria.

Hasnaoui O. 2008. Contribution to the study of the Chamaeropie in the region of Tlemcen. Ecological and mapping. Ph.D. Thesis, University of Tlemcen, Algeria.

Heywood V.H., Brummitt R.K., Culham A., Seberg O. 2007. Flowering Plant Families of the World. Kew Publishing, London. 424 p.
Khatibi R., Soltani S., Khodagholi M. 2016. Bioclimatic classification of central Iran using multivariate statistical methods. Appl. Ecol. Environ. Res. 14: 191-231.

Le Houerou H.N. 1979. Woody forage in North Africa: current state of knowledge. In: Le Houerou H.N. (ed) International Centre for Livestock in Africa Addis Abeba, pp. 57-67.

Le Houerou H.N.1995. Bioclimatology and Biogeography of the Arid Steppes of Northern Africa. Biological Diversity, Sustainable Development and Desertification. Mediter. Opt. Ser. B Res. Stud. 369 p.

Loisel R., Gamila H. 1993. Translation of the effects of brushwood on forest and pre-forest ecosystems by disturbances index. Annals of the Society of Natural Sciences and Archaeology of Toulon and the Var 45: 123-132.

Madon O., Médail F. 1997. The ecological significance of annuals on Mediterranean grassland (Mt Ventoux, France). Plant Ecol. 129: 189-199.

Mankolli H., Cekani M., Abazi U., Kopali A. 2009. Bioclimatic classifications in the ecosystem of Dajt-Tirana, Albania. J. Int. Environ. Appl. Sci. 4:119-124.

Merzouk A. 2010. Contribution to the phytoecological and biomorphological study of halophilic plant populations in the Oran region (Algeria). Ph.D. Thesis, University of Tlemcen, Algeria.

Miara M.D., Ait Hammou M., Hadjadj Aoul S., Dahmani W., Negadi M., Rebbas K., Bounar R., Smaili T. 2016. Note on therophytes in the mountains of Tiaret (massive of GuezoulWestern Algeria). Environ. Ecol. 12: 17-24.

Pouget M. 1980. Soil-vegetation relations in the Southern-Algiers region steppes. Ph.D. Thesis, Aix-Marseille III University.

Ozenda P. 1983. Sahara Flora. CNRS, Paris. 622 p.

Quezel P., Santa S. 1962-1963. New Fflora of Algeria and Southern Desert Regions. C.N.R.S., Paris. 1170 p.

Quezel P.J., Gamisans J., Gruber M. 1980. Biogeography and etablishment of Mediterranean flora. Naturalia Monspelientsia, Montpellier. Pp. 41-51.

Quezel P. 1983. Flora and current vegetation of North Africa, their significance according tothe origin of the evolution and migrations of flora and past vegetation structures. Bothalia 14 : 411-416.

Quezel P., Barbero M. 1990. Mediterranean forests, problems posed by their ecological significance and conservation. Acta Bot. Malac. 15: 145-178.

Quezel P. 2000. Reflection on the Evolution of Flora and Vegetation in Mediterranean Maghreb. Ibis Press, Paris. 118 p.

Raunkiaer C. 1934. The Life Form of Plants and Statistical Plant Geography. Clarendon Press, London. 632 p.

Rivas-Martinez S. 1993. Bases for a new bioclimatic classification of the earth. Folia Bot. Madriet. 10: 1-23.

Rivas-Martínez S. 2004. Global bioclimatics (bioclimatic classification of the earth). Retrieved from http://www. globalbioclimatics.org/book/bi oc/global-Bioclimatics-0.htm.

Rivas-Martínez S. 2008. Global bioclimatics (bioclimatic classification of the earth). http://www.globalbioclimatics. org/book/bioc/global-bioclimatics2008-00.htm.

Rivas-Martinez S., Penas A., Diaz T.E. 2004a. Bioclimatic map of Europe-Bioclimates. Retrieved from http://www. globalbioclimatics.org/form/bi-med.htm.

Rivas-Martinez S., Penas A., Diaz T.E. 2004b. Bioclimatic map of Europe-Thermotypes. Retrieved from http://www. globalbioclimatics.org/form/tb-med.htm.

Rivas-Martínez S., Sáenz S.R., Penas A. 2011. Worldwide 
bioclimatic classification system. Global Geobot. 1: 1-634.

Rivas-Martínez S., Penas A., Del Rio S., Rivas Sáenz S., GarcíaSancho L. 2015. New bioclimatic Antarctic data variants and thermotypes. Int. J. Geobot. Res. 5: 61-63.

Sari Ali A., Benabadji N., Ghezlaoui B.E., Bouazza M. 2011. Physignomic aspects of the halo-resistant and halophilic vegetation in Northern and Southern Western Algeria.
Mediter. Ser. Estud. Biol. 22: 213-250.

Sari Ali A., Benabadji N., Bouazza A. 2012. Floristic composition of the halophilic and salt-resistant plant population in Hammam-Boughrara (Oran-Algeria). Open J. Ecol. 2: 96-108. Villar J.L., Alonso M.Á., Juan A., Crespo M.B. 2014. The genus Tamarix (Tamaricaceae) in Crete (Greece). Willdenowia 44: 321-326. 